\title{
25 Research Square \\ The Effectiveness of a Balanced Salt Solution on Dry Eyes in Comatose Patients: A Randomized Trial
}

Hengameh sadeghi-hasanvand

Lorestan University of Medical Sciences

Parastou Kordestani-Moghadam ( $\square$ kparastou@yahoo.com )

Emergency And Critical Care Department, School Of Nursing And Midwifery, Lorestan University Of

Medical Science https://orcid.org/0000-0003-1251-2516

\section{Farnoosh irandoost}

Lorestan University of Medical Sciences

Seyed hadi ali

Lorestan University of Medical Sciences

Tahereh Toulabi

Lorestan University of Medical Sciences

Farzad Ebrahimzadeh

Lorestan University of Medical Sciences

Fardin heidari

Lorestan University of Medical Sciences

\section{Research note}

Keywords: Balanced Salt Solution, Artificial Tears, Dry Eye, Comatose Patients

Posted Date: March 31st, 2021

DOI: https://doi.org/10.21203/rs.3.rs-362851/v1

License: (c) (i) This work is licensed under a Creative Commons Attribution 4.0 International License.

Read Full License 


\section{Abstract}

Objective: Dry eye is one of the complications of hospitalization in ICUs. This study was conducted to assess the effectiveness of a Balanced Salt Solution (BSS) on dry eyes in comatose patients. This clinical trial was conducted in 2019 on 34 patients admitted to the ICUs. The control group received sterile eye ointment with artificial tears (2 drops every 6 hours) and a tape over the eye. The trial group also received BSS ( 2 drops every 6 hours) in the opposite eye over five days. The patients' dry eye was examined on first day and sixth day of the study using Schirmer's Test (ST) and the Tear Breakup Time Test (TBUT). The data were analyzed in SPSS-21.

Results: The results of the paired T-test based on ST and TBUT showed no significant change from first day one to sixth day in the BSS-receiving group ( $P>0.999$ and $P=0.187$, respectively). Furthermore, no significant difference was observed between the two groups in the mean score of dry eye after eliminating the effect of the demographic and clinical variables $(P=0.947)$. The administration of this solution by nurses seems to be an effective method for preventing the progression of dry eye in ICU patients.

\section{Introduction}

Intensive Care Units (ICUs) provide the most critically-ill patients with care and treatment using advanced equipment [1]. The Patients admitted to ICUs are exposed to a condition called the dry eye [2, 3]. Mercieca et al. found that $75 \%$ of patients in ICUs develop lagophthalmos and are exposed to dryness of the cornea [4]. The results of a study conducted by Masoudi-Alavi et al. on patients admitted to ICUs in Kashan showed that $33.2 \%$ of these patients suffered from dry eye on their fifth day of admission [5]. The method currently used in Iran for patients with closed eyes is to wash the hands before applying eye care and using artificial tear drops [6]. Many studies have shown that artificial tear improves visual acuity [7, 8] and contrast sensitivity [7] in patients with dry eyes. One of the rinsing solutions used is the Balanced Salt Solution (BSS), which is a sterile isotonic physiological solution containing the electrolytes needed for the cells' normal metabolic activities. The results of a study on patients who had underwent phacoemulsification showed that BSS has more advantages than Ringer's lactate solution, such as corneal thickness and spark in the BSS group [9].

Thus, given the high prevalence of dry eyes in ICU patients and the importance of maintaining corneal health in the ICU and the lack of studies on the effectiveness of other eye care methods, including the use of BSS, the present study was conducted to assess the effectiveness of BSS on dry eyes in comatose patients.

\section{Methods}

This study adheres to CONSORT guidelines. The present triple-blind, controlled, clinical trial was conducted on 34 patients gradually admitted to the ICU, from 2019 to 2020 . The study inclusion criteria 
were: Hospitalization in the ICU, mechanical ventilation support in the first 24 hours of admission, the consent of the patient's legal guardian to take part in the study, age 18-75 years, no known history of eye disease, liver disease, and eye surgery in the last six months, GCS score $\leq 8$. The study exclusion criteria were: Less than five days of hospitalization, GCS exceeding 8 in the first three days of hospitalization, spontaneous blinking due to changes in the consciousness level, getting extubated, and the lack of consent on the part of the patient's legal guardian to continue the treatment. Based on $\beta=0.2, a=0.05$, $S 1=1.5, S 2=1.5$ and the below equation, the sample size was determined as 36 .

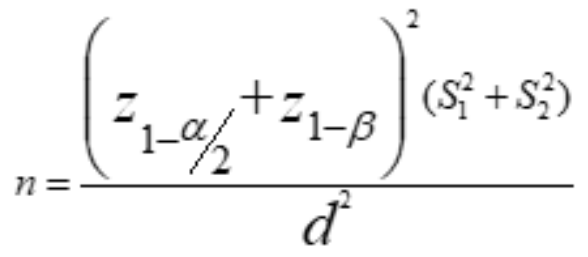

Ongoing sampling was carried out and each eye was then assigned to one of the treatment protocols by the toss of a coin (simple random allocation) perfarmed by nurses.

\section{Study condition}

The patients who met the inclusion criteria entered the study based on the ophthalmologist's opinion and after obtaining written consent from their legal guardian.

Before beginning the intervention, the researcher and ophthalmologist trained the collaborating nurses on how to provide eye care in a uniform manner, and their performance was tested on the patients to ensure consistency. After receiving this practical training on how to provide eye care consistently, these ICU nurses were assigned the duty of providing eye care to the patients as per the given protocols. The eligible patients were then selected for the study. At the outset, ST (to assess their dry eye) and TBUT were carried out by the optometrist. Schirmer Tear Test Strips (made by ERC in Turkey) were used for the ST. Then, each eye was administered one of the eye solutions (either BSS or artificial tear drops) for five days. The eye care protocol in the control group included using two drops of artificial tears every six hours alongside sterile Vitamin A eye ointment applied at the same intervals and the eyes were then covered with tape. In addition to the routine care protocol, the trial group also received BSS (2 drops every 6 hours) for the opposite eye. This solution was prepared and encoded by the Drug Company in the same form as the artificial tear drops. On sixth day, TBUT and ST were performed for both eyes. The required information was collected and entered into the demographic and medical history form by the nurses who had received the initial training. The nurses, the optometrist, and the statistician had no knowledge of the type of eye care solution used until the data analysis was over.

\section{Measurement of the outcomes}

In the present study, no complication was observed after using artificial tears and the BSS. The main outcome of this study was changes in dry eyes, which were assessed through the ST and TBUT on sixth 
day of the intervention, as follows:

In ST, the eye was carefully dried, and then Whatman filter paper strips were bent by $5 \mathrm{~mm}$ from one end and placed in the lateral third of the lower fornix, and after 5 minutes, the length of strip wetted by tears was read in millimeters. A wetted area of $15 \mathrm{~mm}$ or more was considered normal and less than $15 \mathrm{~mm}$ abnormal (1-5 severe, 5-10 moderate, and 10-15) [5]. To measure TBUT, a drop of sodium fluorescein was first poured onto the lower part of the bulbar conjunctiva, and the patient's eyes were opened and closed several times by the examiner so as to spread fluorescein thoroughly in the eye and cover the cornea. Then, a broad ray of blue light was emitted from the ophthalmoscope, and the tear film was assessed without the patient blinking. Black spots or lines indicated dry areas. The time of appearance of the first dry spots was measured in seconds.

The TBUT was interpreted as follows: Duration of time $\leq 5$ seconds = severe; $\leq 10$ seconds = moderate; and $>10$ seconds $=$ mild abnormality [10].

\section{Statistical analysis}

Mean and standard deviation were used to analyze the study data for quantitative variables. Paired t-test was used for in-group comparison in each group and for comparing the mean changes of dry eye between the two groups, a marginal model with GEE estimation method was used through SPSS21 statistical software. A value of $P<0.05$ was considered statistically significant.

\section{Results}

\section{Participant characteristics}

Thirty-four subjects entered the stage of final analysis (A flowchart of the participants can be found in Additional file 1$)$. The majority of the participating patients were male $(67.65 \%)$, had multiple traumas (29.41\%), and were under Assisted mode ventilation (79.41\%). Table 1 presents the other clinical details of the patients. 
Table 1

Description of the clinical status of the participating patients

\begin{tabular}{|c|c|}
\hline Variable & mean \pm standard deviation \\
\hline Tidal volume & $492.06 \pm 46.30$ \\
\hline $\mathrm{FiO}_{2}^{*}$ & $45.59 \pm 11.20$ \\
\hline respiratory rate per minute & $10.71 \pm 1.29$ \\
\hline Oxygen saturation & $96.50 \pm 1.69$ \\
\hline MAP (millimeters of mercury) ${ }^{* *}$ & $90.91 \pm 11.87$ \\
\hline level of consciousness( first day) & $5.09 \pm 1.76$ \\
\hline level of consciousness ( sixth day) & $5.29 \pm 1.92$ \\
\hline Change of level of consciousness & $0.21 \pm 1.37$ \\
\hline \multicolumn{2}{|l|}{${ }^{*}$ The fraction of inspired oxygen } \\
\hline 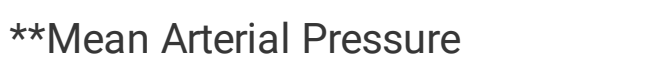 & \\
\hline
\end{tabular}

The results of the paired t-test based on ST and TBUT showed no significant change from first day to sixth day in the BSS group $(P>0.999$ and $P=0.187$, respectively). The results of the paired $t$-test based on ST and TBUT also showed no significant change from first day to sixth day in the artificial tear group ( $P$ $=0.818$ and $\mathrm{P}=0.535$, respectively). Moreover, no significant difference was observed between the two groups in terms of the mean dry eye score after eliminating the effect of the demographic and clinical variables $(P=0.947$; Tables 2 and 3$)$. 
Table 2

Comparison of Schirmer's test score between the intervention and control groups on the first and sixth days of hospitalization

\begin{tabular}{|c|c|c|c|c|}
\hline Group & $\begin{array}{l}\text { Control (artificial } \\
\text { tears) }\end{array}$ & \multirow[t]{3}{*}{$\begin{array}{l}\text { Intragroup p- } \\
\text { value }^{\star}\end{array}$} & $\begin{array}{l}\text { Intervention } \\
\text { (balance solution) }\end{array}$ & \multirow[t]{3}{*}{$\begin{array}{l}\text { Intragroup p- } \\
\text { value }^{*}\end{array}$} \\
\hline \multirow[t]{2}{*}{ day } & $\begin{array}{l}\text { Mean } \pm \text { standard } \\
\text { deviation }\end{array}$ & & $\begin{array}{l}\text { Mean } \pm \text { standard } \\
\text { deviation }\end{array}$ & \\
\hline & In millimeters & & In millimeters & \\
\hline First day & $6.97 \pm 3.78$ & \multirow[t]{2}{*}{0.818} & $6.85 \pm 3.41$ & \multirow[t]{2}{*}{$>0.999$} \\
\hline fifth day & $6.91 \pm 2.99$ & & $6.85 \pm 2.80$ & \\
\hline Score difference & \multicolumn{2}{|l|}{$-0.06 \pm 1.48$} & $0.00 \pm 1.52$ & \\
\hline $\begin{array}{l}\text { Adjusted P-value } \\
\text { between group }\end{array}$ & \multicolumn{2}{|l|}{0.947} & & \\
\hline $\begin{array}{l}\text { Non-Adjusted P-value } \\
\text { between group }\end{array}$ & \multicolumn{2}{|l|}{0.908} & & \\
\hline \multicolumn{5}{|c|}{ *Paired t-test, Intervention: $\mathrm{t} \sim 0.000, \mathrm{df}=33$, Control: $\mathrm{t}=0.232, \mathrm{df}=33$} \\
\hline \multicolumn{5}{|c|}{$\begin{array}{l}{ }^{\star} \text { Generalized linear model (Identity link function) working correlation matrix structure with } \\
\text { Generalized Estimating Equations (GEE), }\end{array}$} \\
\hline
\end{tabular}


Table 3

Comparison of TBUT test score between the intervention and control groups on the first and sixth days of hospitalization

\begin{tabular}{|c|c|c|c|c|}
\hline Group & $\begin{array}{l}\text { Control (artificial } \\
\text { tears) }\end{array}$ & \multirow[t]{3}{*}{$\begin{array}{l}\text { intragroup p- } \\
\text { value }^{\star}\end{array}$} & $\begin{array}{l}\text { Intervention } \\
\text { (balance solution) }\end{array}$ & \multirow[t]{3}{*}{$\begin{array}{l}\text { intragroup p- } \\
\text { value }^{*}\end{array}$} \\
\hline \multirow[t]{2}{*}{ day } & $\begin{array}{l}\text { Mean } \pm \text { standard } \\
\text { deviation }\end{array}$ & & $\begin{array}{l}\text { Mean } \pm \text { standard } \\
\text { deviation }\end{array}$ & \\
\hline & In millimeters & & In millimeters & \\
\hline First day & $5.15 \pm 2.31$ & \multirow[t]{2}{*}{0.535} & $5.24 \pm 2.24$ & \multirow[t]{2}{*}{0.178} \\
\hline fifth day & $5.03 \pm 1.83$ & & $4.88 \pm 1.72$ & \\
\hline Score difference & \multicolumn{2}{|l|}{$-0.12 \pm 1.09$} & $-0.35 \pm 1.50$ & \\
\hline $\begin{array}{l}\text { Adjusted P-value } \\
\text { between group }\end{array}$ & \multicolumn{2}{|l|}{0.142} & & \\
\hline $\begin{array}{l}\text { Non-Adjusted P-value } \\
\text { between group }\end{array}$ & \multicolumn{2}{|l|}{0.129} & & \\
\hline \multicolumn{5}{|c|}{ *Paired t-test, Intervention: $\mathrm{t}=1.376, \mathrm{df}=33$, Control: $\mathrm{t}=0.627, \mathrm{df}=33$} \\
\hline \multicolumn{5}{|c|}{$\begin{array}{l}\star \star \text { Generalized linear model (Identity link function) working correlation matrix structure with } \\
\text { Generalized Estimating Equations (GEE), }\end{array}$} \\
\hline
\end{tabular}

\section{Discussion And Conclusion}

The results showed no significant differences between the artificial tear and BSS groups in terms of the mean scores of ST and TBUT after eliminating the effect of the demographic and clinical variables. The researchers' review of literature showed no studies comparing the effects of BSS and artificial tear drops on dry eyes in comatose patients admitted to ICUs. Vasavada et al. (2009) showed that using BSS had more advantages than Ringer's lactate in terms of corneal thickness and post-operative inflammation in the first day after operation [9]. In agreement with these findings, in another study on intraocular and extraocular tissues, conjunctival and iris tissue samples from patients undergoing cataract surgery and samples from laboratory rabbits, Merrill et al. (1960) found that using BSS for both human and laboratory samples causes no damage to the intraocular tissues compared to sodium chloride [11]. In a clinical trial on patients with dry eyes, Qui et al. (2012) found that the patients' symptoms were relieved after using each of the two care protocols (using punctual plugs vs. artificial tears) and no significant difference was observed between the two groups. In ST, no significant change was observed in the artificial tear group, but the punctual plug group showed significant changes [12]. It can be inferred from the results of the cited studies that other treatment methods are preferable over artificial tears in preventing dry eyes in patients admitted to ICUs. On the one hand, artificial tears normally contain chemical preservatives for preventing bacterial infections and buffers (such as bicarbonate and phosphate) for maintaining the $\mathrm{pH}$ within the normal range (7.4) [13]. On the other hand, the frequent use 
of eye drops containing preservatives, especially benzalkonium chloride (such as gel tears), is associated with ocular allergies and toxicity [14]. Therefore, BSS can be used as an alternative to artificial tears in patients with dry eyes or those exposed to its risk.

The results of the paired t-test based on ST and TBUT showed no significant differences between first days and sixth day in the BSS and artificial tear groups; however, five days after admission, the severity of dry eyes did not worsen compared to first day of admission and it remained within the moderate range.

BSS contains electrolytes needed for the long-term maintenance of endothelial cells, including Sodium Chloride, Potassium Chloride, Calcium Chloride Dihydrate, Magnesium Chloride Hexahydrate, Sodium Acetate Trihydrate, Sodium Citrate Dihydrate, and Sodium Hydroxide [9, 15]. BSS also appears to act as a nutritional supplement. Compared to other solutions, BSS has isotonic osmolality and is neutral (Osm = $302 \mathrm{mMol}, \mathrm{pH}=7.4)$, which is similar to the osmolality of the eye $(0 \mathrm{sm}=302 \mathrm{mMol}, \mathrm{pH}=7.4)$. Moreover, BSS contains a citrate acetate and magnesium buffer system that is essential for Mg-ATPase endothelial pump [9].

As a result, despite the high effectiveness of the noted treatment options, since one of the items determining the ease of use of a drug is its cost-effectiveness, BSS appears to be superior. The results of many studies have shown that using artificial tear improves visual acuity $[7,8]$, and wavefront aberrations [16] in patients with dry eyes. Wei et al. (2020) found that the standard deviation of the objective scatter index (SD-OSI) and the objective scatter index decreased significantly five minutes after the administration of artificial tears in the intervention group of patients with severe dry eyes, while a significant increase was observed in the control group. Nonetheless, 30 minutes after the intervention, no significant difference was observed between two groups in the visual quality parameters. Overall, artificial tears were found to have different effects on the visual quality of patients with dry eyes depending on the severity of their condition [17]. In another systematic study, Ribeiro et al. (2019) found that artificial tears are more effective than ocular lubricants [18]. However, Ishioka et al. (2009) found no significant change in visual acuity in patients with mild dry eyes five minutes after the administration of different concentrations of artificial tears [19].

Based on our findings, BSS can prevent the progression of dry eyes as well as artificial tears. Thus, given the high prevalence of dry eyes among ICU patients and considering the lower costs and good effectiveness of the BSS and its simple formulation, which enables its easier manufacturing, and also the fairly high costs of artificial tear drops, BSS is recommended to be administered by nurses as a useful and effective method for preventing dry eyes in ICU patients.

\section{Limitations}

Given the small sample size of this study and the impossibility of assessing the effects of each of these methods on different severities of dry eyes, further studies with larger sample sizes and on patients with different degrees of dry eyes are required to ensure the generalizability of these findings. 


\section{Abbreviations}

ICUs: Intensive Care Units

BSS: Balanced Salt Solution

TBUT: Tear Break Up Time

ST: Schirmer's Test

\section{Declarations}

\section{Acknowledgements}

The authors hereby wish to express our gratitude to the research deputy of Lorestan University of Medical Sciences for issuing the research ethics code, the nurses working in the ICUs of the select hospital affiliated to this university, and also all the patients and families who helped carry out this study

\section{Author' contributions}

P-K contributed to acquisition and analysis of data. H-S and T-H contributed to study conception and design, analysis and interpretation of data and drafting manuscript. F-E and F-I contributed to study conception and design. F-H and S-H-A contributed to study conception and design and acquisition of data. All authors read and approved the final manuscript.

\section{Funding}

This study was supported by Vice-Chancellor of Research and Technology of Lorestan University of Medical Sciences. Funder had no role in the design of the study and collection, analysis, and interpretation of data and in writing the manuscript.

\section{Availability of data and materials}

According to the memorandum No. 7-147/98/447 dated 20/07/2019 (through Lorestan University of Medical Sciences); The data has been taken from the ICU patients of Shohadaye Ashayer Hospital, affiliated to Lorestan University of Medical Sciences and analyzed. The data is available upon the request from the authors.

\section{Ethic approval and consent to participate}


The present study was conducted in accordance with the Helsinki Declaration guidelines. Approval was obtained from the ethics committee of Lorestan University of Medical Sciences (Code:

IR.LUMS.REC.1398.025) and the study was registered at the Iranian Registry of Clinical Trials (Code: IRCT20200113046121N1). Informed written consent was obtained from the guardians of all the patients before beginning sampling.

\section{Consent to publish}

Not applicable.

\section{Competing interests}

The authors state that they have no competing interests.

\section{References}

1. Patel J, Kurman J, Al-Ghandour E, Thandra K, Mawari S. Predictors of 24-h mortality after interhospital transfer to a tertiary medical intensive care unit. Journal of the Intensive Care Society. 2018;4(2):1-10.https://doi.org/.1177/1751143718765412.

2. Friedman NJ. Impact of dry eye disease and treatment on quality of life. Current opinion in ophthalmology. 2010;21(4):310-6.

3. Rosenberg J, Eisen L. Eye care in the intensive care unit: narrative review and meta-analysis. Critical care medicine. 2008;36(12):3151-5. https://doi.org/10.1097/CCM.0b013e31818f0ee7.

4. Mercieca F, Suresh P, Tullo A. Ocular surface disease in intensive care unit patients. Eye. 1999;13(2):231-40. https://doi.org/10.1038/eye.999.57.

5. Masoudi Alavi N, Sharifitabar Z, Shaeri M, Adib Hajbaghery M. An audit of eye dryness and corneal abrasion in ICU patients in Iran. Nursing in critical care. 2014;19(2):73-7.

6. Davoodabady Z, Rezaei K, Rezaei R. The impact of normal saline on the incidence of exposure keratopathy in patients hospitalized in intensive care units. Iranian journal of nursing and midwifery research. 2018;23(1):57-67. https://doi: 10.4103/ijnmr.IJNMR_187_16.

7. Huang F, Tseng S, Shih M. Effect of artificial tears on corneal surface regularity, contrast sensitivity, and glare disability in dry eyes1. Ophthalmology. 2002;109(10):1934-40. https://DOI: 10.016/s01616420(02)01136-3.

8. Ridder lii W, Tomlinson A, Paugh J. Effect of artificial tears on visual performance in subjects with dry eye. Optometry and Vision Science. 2005;82(9):835-42. https://DOI:

10.1097/01.opx.0000177803.74120.6f.

9. Vasavada V, Vasavada V, Dixit N, Raj S, Vasavada A. Comparison between Ringer's lactate and balanced salt solution on postoperative outcomes after phacoemulsfication: A randomized clinical 
trial. Indian journal of ophthalmology. 2009;57(3):191-9. https://doi:10.4103/0301-4738.49392.

10. Kallarackal G, Ansari E, Amos N, Martin J, Lane C, Camilleri J. A comparative study to assess the clinical use of Fluorescein Meniscus Time (FMT) with Tear Break up Time (TBUT) and Schirmer's tests (ST) in the diagnosis of dry eyes. Eye. 2002;16(5):594-600.

11. Merrill D, Fleming T, Girard LJ. The effects of physiologic balanced salt solutions and normal saline on intraocular and extraocular tissues. American journal of ophthalmology. 1960;49(5):895-8.

12. Qiu W, Liu Z, Zhang Z, Ao M, Li X, Wang W. Punctal plugs versus artificial tears for treating dry eye: a comparative observation of their effects on contrast sensitivity. Journal of ocular biology, diseases, and informatics. 2012;5(1):19-24.

13. Pucker A, Ng S, Nichols J. Over the counter (OTC) artificial tear drops for dry eye syndrome. Cochrane Database of Systematic Reviews. 2016;3(2):145-62. https://doi.org/10.1002/14651858.CD009729.pub2.

14. Tong L, Petznick A, Lee S, Tan J. Choice of artificial tear formulation for patients with dry eye: where do we start? Cornea. 2012;31(3):S32-S6. https://DOI: 10.1097/ICO.0b013e318269cb99.

15. Micromedex M. Balanced Salt Solution. https://www.drugs.com/pro/balanced-salt-solution.html uNITEdstates2018.

16. Montés-Micó R, Cáliz A, Alió JL. Wavefront analysis of higher order aberrations in dry eye patients. Journal of Refractive Surgery. 2004;20(3):243-7.

17. Wei Z, Su Y, Su G, Baudouin C, Labbé A, Liang Q. Effect of artificial tears on aqueous deficient dry eye, a dynamic optical study. BMC Ophthalmology. 2020;3(1):13-22. https://DOI:10.21203/rs.3.rs773/v1.

18. Ribeiro MVMR, Barbosa FT, Ribeiro LEF, Sousa-Rodrigues CFd, Ribeiro EAN. Effectiveness of using preservative-free artificial tears versus preserved lubricants for the treatment of dry eyes: a systematic review. Arquivos brasileiros de oftalmologia. 2019;82(5):436-45.

19. Ishioka M, Kato N, Takano Y, Shimazaki J, Tsubota K. The quantitative detection of blurring of vision after eyedrop instillation using a functional visual acuity system. Acta ophthalmologica. 2009;87(5):574-5. https://DOI: 10.1111/j.755-3768.2008.01230.x.

\section{Supplementary Files}

This is a list of supplementary files associated with this preprint. Click to download.

- CONSORT2010Checklist.doc

- DIA.docx 